\title{
CHEMICAL NONEQUILIBRIUM EFFECTS IN THE WAKE OF AN OBSTACLE IN HYPERSONIC BOUNDARY-LAYER FLOWS
}

\section{Birrer and C. Stemmer}

\author{
Lehrstuhl für Aerodynamik \\ Technische Universität München \\ Garching b. München D-85748, Germany
}

\begin{abstract}
Various complex flow features occur in reentry scenarios. This paper aims to investigate the influence of chemical nonequilibrium effects on the wake of a cuboid obstacle, sized about half the boundary layer thickness, in hypersonic boundary layer flows. The geometry here was chosen according to NASA HyBoLT experiment as explained in [1], where the basic shape is a wedge with a half angle of $6^{\circ}$. The freestream flow was chosen to have atmospheric conditions at an altitude of $42.5 \mathrm{~km}$ and a Mach number of 8.5. Simulations were obtained with 3 different gas models, a caloric one, one assuming chemical equilibrium, and the last considering chemical nonequilibrium. Two-dimensional (2D) direct numerical simulation (DNS) was used over the whole domain to extract inflow profiles for three-dimensional (3D) DNS of a reduced domain.
\end{abstract}

\section{INTRODUCTION}

Returning space travelling vehicles are always exposed to extreme heat loads during reentry. The very high velocities can cause temperatures of several thousand Kelvin. In these conditions, complicated mechanisms such as dissociation, ionization, and excitation of vibrational degrees of freedom start gaining influence on the flow behavior.

Postflight investigations on returned capsules have shown that around the stagnation point, the flow can be predicted quite well with quasi-stationary methods, while further downstream predictions can show significantly different results to what occurs in reality. Former ways of handling these uncertainties were to assume turbulence over the whole surface and to add a considerable safety factor. Transition to turbulence can increase the heat transfer to the surface by a factor of 3 and demands a much higher heat protection. Therefore, accurate prediction

This is an Open Access article distributed under the terms of the Creative Commons Attribution-Noncommercial License 3.0, which permits unrestricted use, distribution, and reproduction in any noncommercial medium, provided the original work is properly cited. 
of the flow behavior is needed to assure a sufficiently dimensioned heat shield, without overpredicting it.

The influence of surface roughness, meaning surface protrusions that are small compared to the boundary layer thickness, on hypersonic transition has recently been investigated by several groups (see, e.g., [2-4]). It is not possible to match all relevant characteristic scales (in particular, the Damköhler number) in such wind tunnel tests, and free-flight experiments are extremely difficult to achieve under controlled conditions and with accurate measurements. Therefore, numerical investigations give some additional valuable insight.

Receptivity analysis has been done in [5] for ideal gas. This paper focuses on the effect of chemical reactions and nonequilibrium conditions in a hypersonic boundary layer flow over an obstacle.

\section{NUMERICAL DETAILS}

\subsection{Code}

The results shown here were obtained with the NSMB code (Navier-Stokes Multi Block) $[6,7]$. The package uses higher order finite differences schemes with explicit (Runge-Kutta) or implicit time integration on multiple blocks using the MPI (message passing interface) environment. Second-order artificial dissipation terms are added to capture shock waves. Generalized coordinates are used on a body fitted mesh. Various gas models are implemented in the code which allows modeling of hot gas flows.

\subsection{Governing Equations}

The complete compressible Navier-Stokes equations, with three different gas models, are solved. First, a caloric perfect gas model was used; then, chemically reacting air in equilibrium was assumed; and at last, chemical nonequilibrium was taken into account. Here, the chemical reactions were considered with a five species Park [8] model where the species are $\mathrm{N}, \mathrm{NO}, \mathrm{N}_{2}, \mathrm{O}$, and $\mathrm{O}_{2}$. This chemical model is assumed to be valid up to $9000 \mathrm{~K}$. The equilibrium constants for the second model are calculated to minimize the free Gibbs energy. The nonequilibrium model calculates the concentrations by solving 17 reaction equations (three dissociation reactions with any collision partner and two recombination reactions):

$$
\begin{aligned}
\mathrm{N}_{2}+M & \rightleftharpoons \mathrm{N}+\mathrm{N}+M ; \\
\mathrm{NO}+M & \rightleftharpoons \mathrm{N}+\mathrm{O}+M ; \\
\mathrm{O}_{2}+M & \rightleftharpoons \mathrm{O}+\mathrm{O}+M ;
\end{aligned}
$$




$$
\begin{aligned}
\mathrm{N}_{2}+\mathrm{O} & \rightleftharpoons \mathrm{NO}+\mathrm{N} ; \\
\mathrm{NO}+\mathrm{O} & \rightleftharpoons \mathrm{N}+\mathrm{O}_{2} .
\end{aligned}
$$

Here, $M$ stands for any one of the five species.

The caloric model uses Sutherlands law to obtain the viscosity:

$$
\frac{\mu}{\mu_{\infty}}=\left(\frac{T}{T_{\infty}}\right)^{3 / 2} \frac{T_{\infty}+S_{1}}{T+S_{1}}
$$

Here, $\mu_{\infty}$ represents the viscosity at the reference temperature $T_{\infty}$ and the parameter $S_{1}=110.3 \mathrm{~K}$.

The two models considering chemistry use the Blottner model to calculate the viscosity and thermal conductivity of each species [9]:

$$
\mu_{s}=0.1 \exp \left[\left(A_{s} \ln (T)+B_{s}\right) \ln (T)+C_{s}\right] .
$$

The mixture viscosity is calculated by Wilke's semiempirical relation [10]:

$$
\mu_{\operatorname{mix}}=\sum_{s} \frac{X_{s} \mu_{s}}{\sum_{s} X_{r} \phi_{s r}}
$$

where

$$
\Phi_{s r}=\frac{1}{\sqrt{8}}\left(1+\frac{M_{s}}{M_{r}}\right)^{-1 / 2}\left[1+\left(\frac{\mu_{s}}{\mu_{r}}\right)^{1 / 2}\left(\frac{M_{r}}{M_{s}}\right)^{1 / 4}\right]^{2}
$$

\section{SIMULATIONS}

\subsection{Geometry}

The setup for the simulations was chosen according to the geometry used for the HyBoLT [1] flight experiment, which was launched in 2008, but was aborted, due to the wrong trajectory that the flight vehicle started to take.

The basic shape is a wedge with half angle $6^{\circ}$. The leading edge has a radius of $3.81 \mathrm{~mm}$, which puts it $32.64 \mathrm{~mm}$ behind a virtual sharp leading edge; $508 \mathrm{~mm}$ behind the virtual leading edge surface trips are located. For this work, the authors focus on a cubical obstacle with a height of $8.9 \mathrm{~mm}$, which corresponds to roughly $30 \%$ of the local boundary layer. Span- and streamwise dimensions were set to be two times the height. Figure 1 shows the setup for the $2 \mathrm{D}$ simulations. 


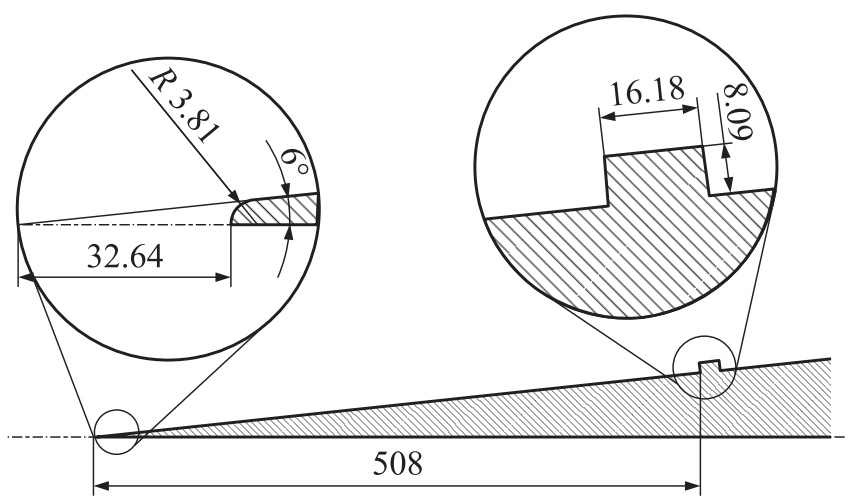

Figure 1 Sketch of the 2D geometry used in the study based on the HyBoLT design [1]. In 3D, the spanwise dimension of the obstacle is equal to the streamwise size. Dimensions are in millimeters

\subsection{Flow Conditions}

It was decided to concentrate on the flow conditions predicted for the last measurement point of the HyBoLT flight experiment [1] at Mach 8.5. Having the highest Mach number, these conditions seemed most promising to notice an influence of the nonequilibrium effects. The freestream temperature and density were calculated using the 1976 Standard Atmosphere at an altitude of $42.5 \mathrm{~km}$. Freestream velocity is set to $2732.6 \mathrm{~m} / \mathrm{s}$ and a Reynolds number of $4.66 \cdot 10^{5}$ was taken.

\subsection{Computational Grids}

Simulations were conducted on two grids. First, 2D calculations on a domain covering the whole geometry for this setup were obtained. These results then delivered appropriate inflow profiles after the bow shock of the leading edge which were used for detailed 3D simulations on a reduced domain around the obstacle. The 2D grids consisted of 16 blocks with a resolution around the obstacle of 16 cells for the height and 32 for the length, leading to a total of $2.1 \cdot 10^{5}$ finite volume cells.

The reduced domain for the $3 \mathrm{D}$ calculations is about 13 times the obstacle length in streamwise direction, 7 times in spanwise, and about 7 times the obstacle height in surface normal direction. Thirty-five blocks with close to 11 million cells were used. The obstacle was resolved with 32 cells for the height and 64 for the span- and streamwise directions. 


\section{RESULTS}

\subsection{Two-Dimensional Results}

Comparing the maximum temperatures of the three gas models shows, as expected, a reduction of the peak values when considering chemistry. Neglecting the zone in front of the bow shock, as it is not the area of interest, a drop from $1913 \mathrm{~K}$ in the caloric case to $1769 \mathrm{~K}$ in the chemical equilibrium case is registered. These temperatures are seen at the top front corner of the obstacle. Figure 2 shows temperature profiles normal to the wall obtained from the 2D calculations. They are extracted at the wall point located $340 \mathrm{~mm}$ behind the virtual leading edge. These are the temperature profiles used as input for the $3 \mathrm{D}$ simulations. As pointed out above, a temperature reduction due to the consideration of chemical reactions is noticable. But while in chemical equilibrium, it seems to be a pure reduction of the temperatures, considering nonequilibrium also results in a shift of the peak value towards the wall. This results in a higher temperature gradient, which is a more critical measure, as it means there are higher heat fluxes into the surface.

Table 1 contains species concentrations for both 2D and 3D simulations. For the reaction products $\mathrm{N}, \mathrm{NO}$, and $\mathrm{O}$ the maximum values behind the bow shock are shown; for $\mathrm{N}_{2}$ and $\mathrm{O}_{2}$, the minimas were inserted. The highest value of a product concentration is $0.47 \%$, showing that the extent of the reactions is quite small. In the nonequilibrium case, the concentrations are even smaller.

Figure 3 shows zero streamwise contours for all models. The recirculation zones obtained with the equilibrium model in the middle is just slightly smaller than the ones of the caloric model at the top. The bottom figure which represents the nonequilibrium model shows significantly reduced recirculation zones.

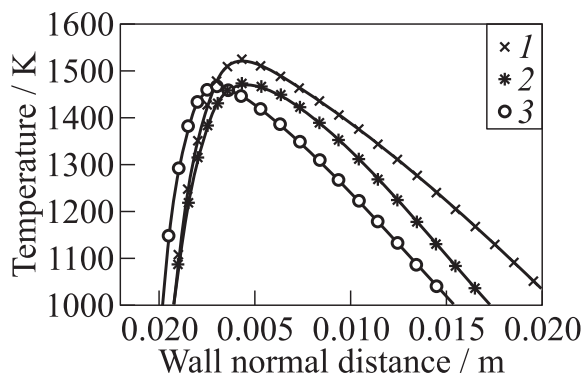

Figure 2 Temperature as a function of the wall normal distance. The data is taken from the $2 \mathrm{D}$ simulations of the different gas models, starting at the surface point at $x=0.34 \mathrm{~m}$ behind the virtual leading edge: 1 - caloric model; 2 - equilibrium; and 3 - nonequilibrium gas models 
Table 1 Species concentrations of the two chemical models from the 2D and $3 \mathrm{D}$ simulations. For the 2D cases, only values behind the shock are considered. For the reaction products $\mathrm{N}, \mathrm{NO}$, and $\mathrm{O}$, the maximal values are given; for $\mathrm{O}_{2}$ and $\mathrm{N}_{2}$, the minimal concentrations are shown

\begin{tabular}{lcccc}
\hline \multirow{2}{*}{ Species } & \multicolumn{2}{c}{ Chemical equilibrium } & \multicolumn{2}{c}{ Chemical nonequilibrium } \\
\cline { 2 - 5 } & $2 \mathrm{D}$ & $3 \mathrm{D}$ & $2 \mathrm{D}$ & $3 \mathrm{D}$ \\
\hline $\mathrm{N}$ & $9.26 \cdot 10^{-11}$ & $1.29 \cdot 10^{-7}$ & $6.87 \cdot 10^{-12}$ & $4.06 \cdot 10^{-10}$ \\
$\mathrm{NO}$ & $4.79 \cdot 10^{-3}$ & $1.70 \cdot 10^{-2}$ & $2.96 \cdot 10^{-7}$ & $1.35 \cdot 10^{-6}$ \\
$\mathrm{~N}_{2}$ & $7.65 \cdot 10^{-1}$ & $7.59 \cdot 10^{-1}$ & $7.67 \cdot 10^{-1}$ & $7.67 \cdot 10^{-1}$ \\
$\mathrm{O}$ & $2.59 \cdot 10^{-4}$ & $7.77 \cdot 10^{-3}$ & $4.15 \cdot 10^{-5}$ & $4.09 \cdot 10^{-5}$ \\
$\mathrm{O}_{2}$ & $2.30 \cdot 10^{-1}$ & $2.16 \cdot 10^{-1}$ & $2.33 \cdot 10^{-1}$ & $2.33 \cdot 10^{-1}$ \\
\hline
\end{tabular}

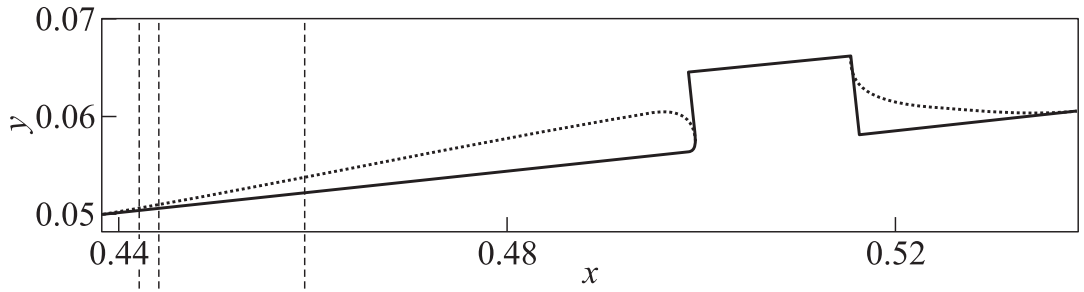

(a)

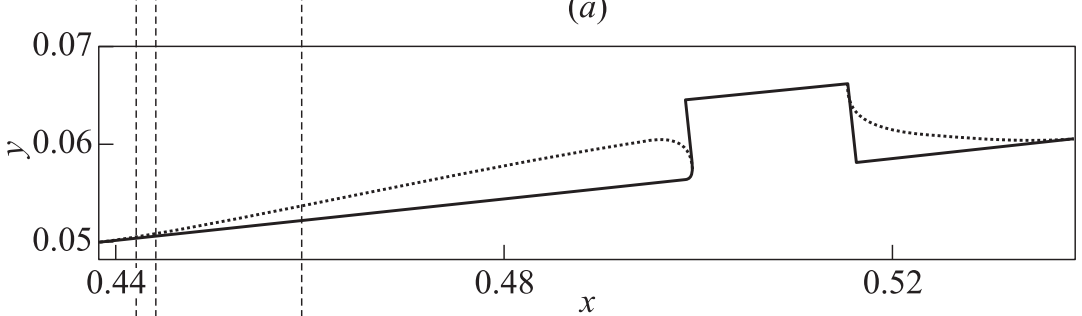

(b)

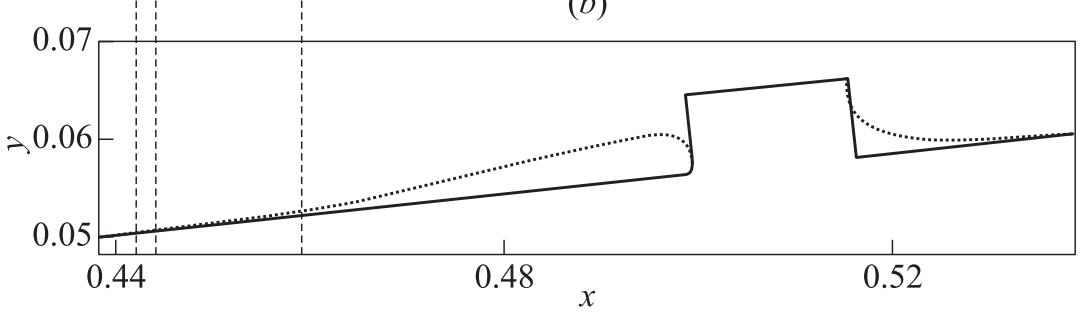

(c)

Figure 3 Zero $u$-velocity contour plots for the three different gas models: $(a)$ caloric case; $(b)$ chemical equilibrium case; and $(c)$ chemical nonequilibrium. The vertical lines were included to mark the begining of the recirculation zones. 


\subsection{Three-Dimensional Results}

The temperature fields of the three models show similar tendencies as in the $2 \mathrm{D}$ cases. Again, assuming equilibrium chemistry reduces the maximal temperature significantly. On the other hand, when chemical reactions in nonequilibrium are considered, the difference of the maximum temperatures, compared to the caloric case is smaller and also, in $3 \mathrm{D}$, there is a shift of the temperature peak towards the wall, which can result in temperature gradients that even exceed those reached in the caloric case. The highest temperatures in the wake of the obstacle, as well as the corresponding locations, are given in Table 2 for the three gas models. As seen in Table 1, reaction products reach slightly higher concentrations than in 2D, but are still small. Due to the proper representation of the horse-shoe vortex in three dimensions, differences of the recirculation zones are no more that significant as in 2D.

Table 2 Temperature maximas in the wake of the obstacle and their locations of the three different gas models. The reference for the locations is the front, lower, and right corner of the obstacle

\begin{tabular}{lcccc}
\hline \multicolumn{1}{c}{ Case } & Temperature, $\mathrm{K}$ & $X, \mathrm{~mm}$ & $\mathrm{Y}, \mathrm{mm}$ & $Z, \mathrm{~mm}$ \\
\hline Caloric & 1860 & 60.2 & 4.42 & 8.22 \\
Equilibrium & 1690 & 63.9 & 3.66 & 7.72 \\
Nonequilibrium & 1810 & 56.6 & 1.15 & 7.46 \\
\hline
\end{tabular}

For the $3 \mathrm{D}$ simulations, the main interest lies in the wake of the obstacle. Figures 4 and 5 show temperature contours of three planes, one for each axis direction. All three contain the point of maximum temperature in the wake. Figure 4 shows the results from the equilibrium case and Fig. 5 from the nonequilibrium case. For both cases, in the $y z$-plane at the top, a mushroom shaped area formed by two counterrotating vortices is visible. It contains two locations with temperature peaks. In the nonequilibrium case, these are also the areas where concentrations of the reaction products reach their maximas, while with chemical equilibrium, the highest concentrations occur in front of the obstacle, where temperatures are highest. The horse-shoe vortex, far outside of the center, seems to play a less significant role in this setup. The distance of the temperature peaks move away from the wall going downstream, as can be seen in the $x z$-plane. The temperature peak in the nonequilibrium case is closer to the wall and the obstacle than in the equilibrium case, causing higher heat transfers in both directions.

\section{CONCLUDING REMARKS}

Two- and three-dimensional DNS were obtained for the HyBoLT experimental geometry. A cubical obstacle located about $500 \mathrm{~mm}$ behind the virtual leading 


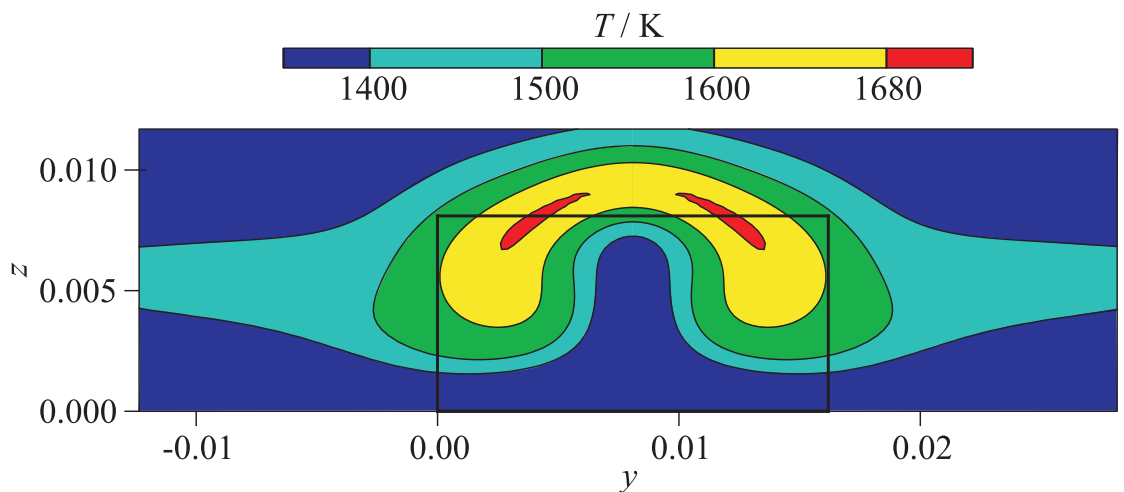

(a)

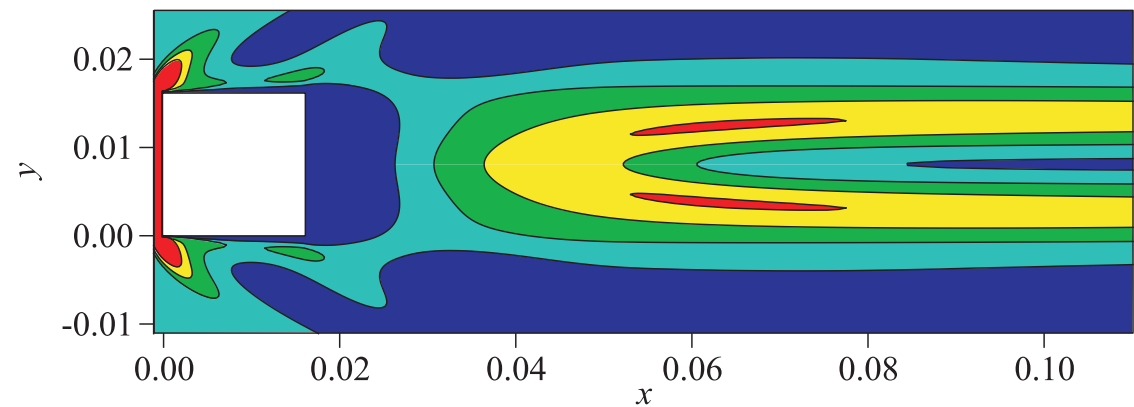

(b)

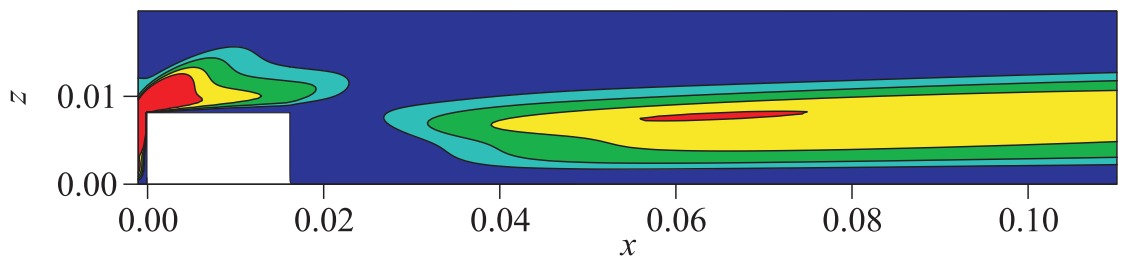

(c)

Figure 4 Contour plots of temperature shown in three planes cutting through the point of maximal temperature in the wake: $(a)$ a slice at constant $x=0.0639 \mathrm{~m} ;(b)$ at constant $z=0.00772 \mathrm{~m}$; and $(c)$ at constant $y=0.00366 \mathrm{~m}$ from the lower front corner. The data are from the case with equilibrium chemistry. The contour lines show the temperatures 1400, 1500, 1600, and 1680 K. (Refer Birrer and Stemmer, p. 274.)

edge creates two counterrotating vortices in the direct wake of the obstacle, pushing hot gases closer to the wall. The horse-shoe vortex does not seem to have the same impact on the heat transfer and, therefore, is not described in detail in this work. 


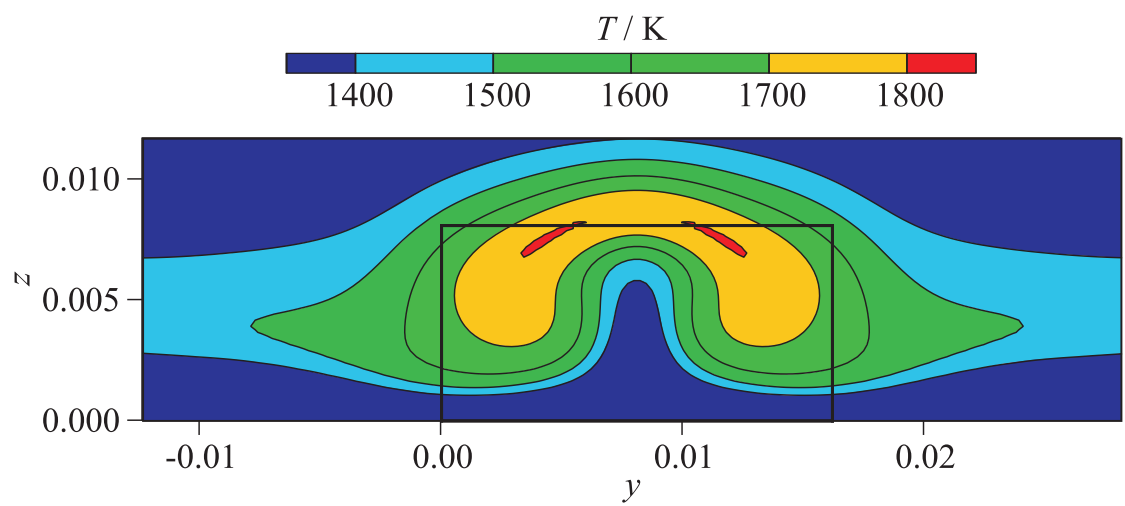

(a)

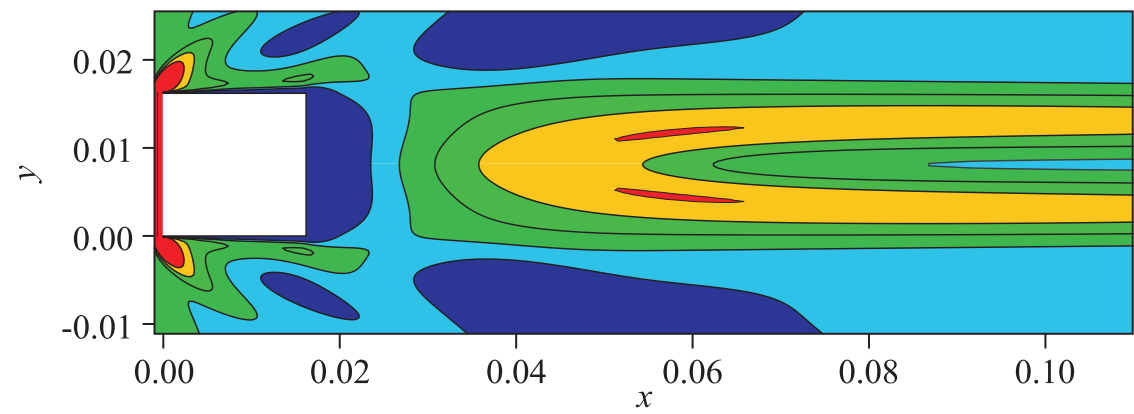

(b)

N

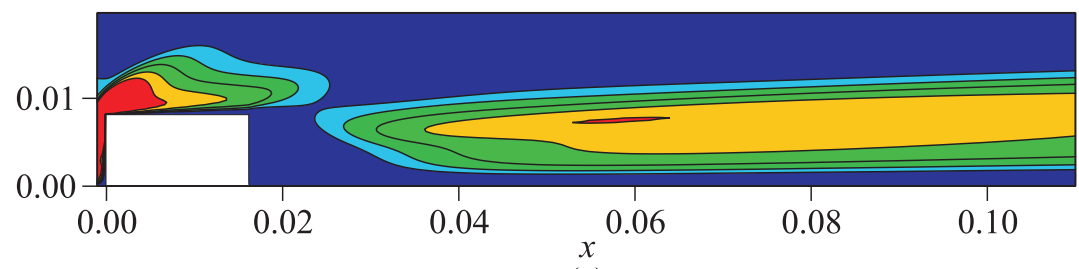

(c)

Figure 5 Contour plots of temperature shown in three planes cutting through the point of maximal temperature in the wake taken at constant $x=0.0566 \mathrm{~m}(a), y$ $=0.00115 \mathrm{~m}(b)$, and $z=0.00746 \mathrm{~m}(c)$. The data are from the case with nonequilibrium chemistry. The contour lines show the temperature from 1400 to $1800 \mathrm{~K}$ in steps of 100 K. (Refer Birrer and Stemmer, p. 275.)

Inflow profiles for the $3 \mathrm{D}$ simulations are extracted from the $2 \mathrm{D}$ solutions. The maximum temperatures for all three gas models considered here, caloric perfect gas, chemical equilibrium, and chemical nonequilibrium, occur at the top front corner of the obstacle. More interesting, regarding heat shield design, is the 
increased heat transfer due to the counterrotating vortices. Highest temperatures of all three cases appear, as expected, in the caloric case. Assuming chemical equilibrium then reduces the temperature considerably. In the nonequilibrium case, the temperature drop is no more that high, but more important is that the temperature peak moves closer to the wall, causing increased temperature gradients and, therefore, higher heat transfer into the surface.

Further investigations for more detailed research of the wake are ongoing and including of thermal nonequilibrium models is planned.

\section{ACKNOWLEDGMENTS}

The authors would like to thank the staff of the Center of Turbulence Research in Stanford, especially O. Marxen and G. Iaccarino for their scientific support.

\section{REFERENCES}

1. Berry, S.A., C. Fang-jenq, M. C. Wilder, and D. C. Reda. 2007. Boundary layer transition experiments in support of the hypersonics program. AIAA Paper No. 2007-4266,

2. Schneider, S. P. 2007. Effects of roughness on hypersonic boundary-layer transition. AIAA Paper No. 2007-305.

3. Berry, S. A., and T. J. Horvath. 2007. Discrete roughness transition for hypersonic flight vehicles. AIAA Paper No. 2007-307.

4. Tirtey, S. C., H. Bolnot, H. Bottini, S. Paris, D. G. Fletcher, and O. Chazot. 2008. In-flight hypersonic roughness induced transition experiment. AIAA Paper No. 2008-250.

5. Wang, X., and X. Zhong. 2008. 2008. Receptivity of a hypersonic flat-plate boundary layer to surface roughness. AIAA Paper No. 2008-503.

6. Vos, J. B., J. Bohbot, S. Champagneux, et al. 2006. NSMB 5.96 User Guide.

7. Vos, J. B., P. Leyland, V. van Kemenade, et al. 2007. NSMB Handbook 4.5.

8. Park, C. 1985. On convergence of chemical reacting flows. AIAA Paper No. 19850427.

9. Blottner, F. G., M. Johnson, and M. Ellis. 1970. Chemically reacting viscous flow program for multi component gas mixture. Sandia Laboratories, SC-RR-70-754.

10. Bird, R. B., W.E. Stewart, and E. N. Lightfoot. 1960. Transport phenomena. New York: John Wiley \& Sons. 